\title{
A study of X-ray volume imaging system in image guided radiotherapy with variable gantry rotations
}

\author{
NVN Madhusudhana Sresty', Krishnam Raju Alluri' ${ }^{1}$, Ramanjappa Thogata ${ }^{2}$, Shabbir Ahmed ${ }^{1}$, \\ Lakshmi Venkataramana Puriparthi' ${ }^{1}$, Ramakrishna Ketham¹, Vijay Kumar Mogulagani1
}

\author{
${ }^{1}$ Department of Radiotherapy, Basavatarakam Indo American Cancer Hospital \& Research Institute, Hyderabad, India \\ ${ }^{2}$ Department of Physics, Sri Krishnadevaraya University, Anantapur, Andhra Pradesh, India
}

Received May 21, 2015; Revised January 05, 2016; Accepted January 06, 2016; Published Online January 9, 2016

\section{Technical Report}

\begin{abstract}
Purpose: The main purpose of this work is to investigate the optimal usage of $\mathrm{X}$-ray volume imaging (XVI) system in image-guided radiotherapy with different gantry rotations in order to reduce scanning volume. Methods: A total of 60 scans of 16 individual patients with breast and head and neck cancer were used in this study. Full and partial gantry rotations were performed at the same time with same setup on the couch using XVI system by changing the preset information. The reference and localization images were matched with this system. The set up errors were evaluated with XVI software. Results: Variation in translational errors with full and half gantry rotations in breast cases were $<2 \mathrm{~mm}$ in $86.6 \%$ of measurements. Similarly, variations between full and partial gantry rotations in head and neck cases were $<1 \mathrm{~mm}$ in $95.5 \%$ of measurements. Results showed almost similar translational and rotational shifts in both full and partial gantry rotations in the majority of the cases. Conclusion: Based on selected cases in this study, partial rotation of the gantry for acquiring 3D cone beam computerized tomography (CBCT) is very useful option in reducing scanning volume and total treatment time in IGRT. However, the use of partial rotation of the gantry depends on patient thickness and area to be reconstructed to track anatomical changes near to the target.
\end{abstract}

Keywords: IGRT; Partial Rotation; XVI

\section{Introduction}

Reproducible positioning of the patient on the couch of a treatment unit for radiation delivery is one of the main requirements for the tumor control in radiotherapy. This can be checked and corrected if necessary as per the prior plan with the imaging systems mounted with the treatment delivery system. ${ }^{1}$ Cone beam computed tomography (CBCT) integrated with linear accelerator for patient position verification is one of the major 3 dimensional (3D) methods in image guidance. ${ }^{2,3}$

Image guided radiotherapy (IGRT) is typically performed with the help of different imaging techniques to obtain patient setup information just before the treatment delivery. If the patient is not properly immobilized and placed on the couch, adjustments are made such that the patient can be treated with minimized setup errors. Kilo-voltage (KV) CBCT is one method in IGRT to localize the target volume based on volumetric information obtained by X-ray volume imaging (XVI) system. Linear accelerators attached with CBCT system are now available for IGRT. A CBCT has a beam, which is wider in patient's longitudinal direction. Cone-beam provides the possibility to make a whole volume scan in one single rotation of the XVI system around the patient in treatment position. Setup errors can be corrected by adjustment of translational and rotational deviations between the reference image and localization image. Planning CT images and CBCT projections are used to form the reference image and localization image, respectively, with XVI software.

CBCT reconstruction ${ }^{4}$ starts simultaneously with image acquisition and ensures the availability of transverse, coronal, and sagittal images immediately at the end of

\section{Corresponding author: NVN Madhusudhana Sresty; Department of Radiotherapy, Basavatarakam Indo American Cancer Hospital \&} Research Institute, Hyderabad, India.

Cite this article as: Sresty NVNM, Alluri KR, Thogata R, Ahmed S, Venkataramana Puriparthi L, Ketham R, Mogulagani VK. A study of $X$-ray volume imaging system in image guided radiotherapy with variable gantry rotations. Int J Cancer Ther Oncol 2016; 4(1):411. DOI: 10.14319/ijcto.41.1 
acquisition. The algorithm used for this image reconstruction is a Feldkamp-type algorithm.5,6,7 The Feldkamp-type algorithm uses an approximation in the Filtrated Back-Projection (FBP) process through a weighting function.5,6,7 The major parts of the algorithm include pre-weighting the projections, and a three dimensional back projection. ${ }^{5,6,7}$ The weighting is mainly based on both the fan beam and the cone beam angles. 5,6,7 Projections are handled row by row and filtering is only implemented in the transverse direction of the sampled projections only. ${ }^{5,6,7}$ In the 3-D backprojection step, all filtered fan-beam projections have to be linearly interpolated along the tilted plane to determine the intensities in each voxel for a particular cone-angle.5,6,7 All these voxel intensities are then summarized to get the complete three dimensional volumes. XVI system has the potential to match three dimensionally, using reference CT images and localization images. 5,6,7 This allows positional correction for translation and rotation which can then be translated into table movements. ${ }^{5,6,7}$ Utilization of CBCT system method made IGRT, a better three-dimensional (3D) set up technique to improve daily patient treatment delivery. $3,8,9,10$

XVI reconstruction is possible with partial gantry rotations. In IGRT, there is a potential of using XVI system on daily basis during total course of treatment to deliver more accurate treatments. The additional dose from the daily use of CBCT could approach deterministic levels. ${ }^{11}$ CBCT may increases secondary cancer risk up to $2-4 \%$ if it is taken daily. ${ }^{12}$ The main aim of this study was to investigate the optimal usage of XVI system to reduce scan volume of the patient and deliver least possible additional dose to the patient with more accurate 3D shift information.

In our study, full and partial gantry rotation methods were selected to acquire CBCT images from XVI system. In a full rotation method, gantry rotates around the patient to a total 360 degrees, whereas in partial rotation method, rotation is as specified in the preset. During gantry rotation, X-ray tube of XVI system at right angle to the gantry is switch on position to obtain continuous CBCT projections. A number of CBCT images acquired (scan volume) are typically more in a full gantry rotation (complete 360 degree rotation) method compared with partial rotation method. If the reconstructed CBCT image obtained with partial rotation gives same setup shifts after matching procedure similar to that of full rotation method with clear visualization of required image, then the aim of this study will be achieved with less scanning volume. Furthermore, 3D localized image is obtained with a partial gantry rotation, and shifts are compared using both methods. Matching procedure is confined to a specific volume which was selected before the scanning process.

\section{Methods and Materials}

Our previous study ${ }^{3}$ described the methods for the optimal usage of XVI system with different field of views. The current study will explain the method with different gantry rotations for the breast and head and neck cancer. For details on methods and materials, readers are advised to refer to our previous study ${ }^{3}$.

In summary, we have used Elekta Synergy (Elekta Oncology Systems Ltd, Crawley, UK) linear accelerator with CBCT for the IGRT. The accelerator has a KV X-ray source (Eureka Rad-92, Varian Sapphire Housing) and amorphous silicon flat panel imagers, both mounted at $90^{\circ}$ to the treatment head. Process of obtaining CBCT image is same in both full rotation and partial rotation methods used in this study except the number of images obtained during gantry rotation. In both methods, images were reconstructed using same algorithm. CBCT projections obtained during full rotation and partial rotation were 650 and 360, respectively. Therefore, the reconstructed volume information was much better in a full rotation. Such reconstructed CBCT volume images were then utilized for the matching. Matching procedure in the software is of major importance. The localized image is shifted to fit the reference CT following proper verification in sagittal, coronal, and transverse planes, and this procedure is called "matching". Registration is carried out by the system on pixels that exist inside the clip box only. The matching for high densities is carried out based on tissue that has 1.5 times the density of water. The Grey value mode of automatic registration takes into account voxel intensity values throughout the entire image volume. The operation of this algorithm may take several minutes to complete the matching process, whereas bone match can be completed within seconds. The third way is to do the manual matching by an expert. .

The XVI system has predefined standard settings for different lesions, and allows full or partial rotations. One can modify these presets depending upon requirement. Researchers examined the CBCT images of breast with partial rotation of 190 degrees and found satisfactory. ${ }^{13}$ XVI standard preset of chest which is defined with full gantry rotation is available as a default. A preset information which allows half rotation $\left(90^{\circ}-270^{\circ}\right)$ of gantry was created in XVI system. Clarity in visualisation of left breast and lung is not required when scanning right breast and vice versa. If we perform a CBCT scan which covers the required breast with half rotation only, this could potentially achieve the aim of the study. Hence, this preset was used for the breast scanning with partial rotation. Elekta standard preset for head and neck was with partial rotation, with 361 projections over an angle of $200^{\circ}\left(260^{\circ}-100^{\circ}\right)$ and S20 collimator. There was no standard preset which allows full rotation for head and neck cases. A preset was created to proceed for full gantry rotation. Specified preset start and stop 
angles were from $181^{\circ}$ to $180^{\circ}$ in all full rotation scans. The $\mathrm{kV}$ and $\mathrm{mAs}$ for breast CBCT were 120 and 650, respectively, in full gantry rotation, whereas 120 and 325 , respectively, in partial rotation as per the preset. Similarly $100 \mathrm{kV}$ and $36.1 \mathrm{mAs}$ were used in head and neck partial rotation scans, whereas $100 \mathrm{kV}$ and $65 \mathrm{mAs}$ for full gantry rotation scans.

Patients were taken to the mould room and immobilized with thermoplastic mask (Orfit industries, Vosveld 9A, Belgium). The scans were taken using full $\left(360^{\circ}\right)$ and partial gantry rotations at the same time by modifying the presets if necessary. A total of 16 different patients were used ( 8 breast cancer and 8 head and neck cancer) for full and partial gantry rotation scans. Breast cases that had lateral separation of less than $35 \mathrm{~cm}$ at center of the target level were selected for partial rotation scans. There were no such limitations in head and neck cases.

The planning CT study set, structure set, and plan of each case were exported from the $\mathrm{XiO}$ treatment planning system (version, 4.33.02; Computerized Medical Systems, St. Louis, USA) to the XVI workstation. The planning isocenter was taken as correction reference point. The translational and rotational shifts were obtained based on this reference. Sub volumes were identified by using alignment clip box to cover the target volume. Previous study ${ }^{14}$ revealed that Grey value matching is superior, and hence, this mode of automatic registration was performed in all cases; thus allowing us to calculate the setup shifts. Although automatic registration method was used for the matching, physician had verified the alignment between both the images using manual matching, and the final patient shifts were implemented.

\section{Results}

This study revealed a number of interesting observations. Figures 1, 2 and 3 are the line graphs of lateral, longitudinal and vertical shifts in breast cases measured in full and partial gantry rotation methods, respectively. In line graph, both the methods are shown by different dots. Vertical lines between the dots in the graph represent difference in shift between two methods. Variation in treatment setup errors with full and partial gantry rotations were $<1 \mathrm{~mm}$ in $68.8 \%$ of measurements and $<2 \mathrm{~mm}$ in $86.6 \%$ of measurements, which included lateral, longitudinal and vertical directions (Figures 1, 2 and 3). Visualisation of chest wall, lung, and heart was enough with partial rotation. Similarly, Figures 4, 5 and 6 are the line graphs in head and neck scans. Variations between full and partial gantry rotation in these lesions were $<1 \mathrm{~mm}$ in $95.5 \%$ of measurements and $<1.2 \mathrm{~mm}$ in $100 \%$ of measurements. Figures 7 and 8 are the localized images obtained in full and partial gantry rotation methods of a case of breast. Maximum variations in rotational shifts were $<2^{0}$ (Table1) in breast scans and $<1^{0}$ (Table2) in head and neck scans, respectively.

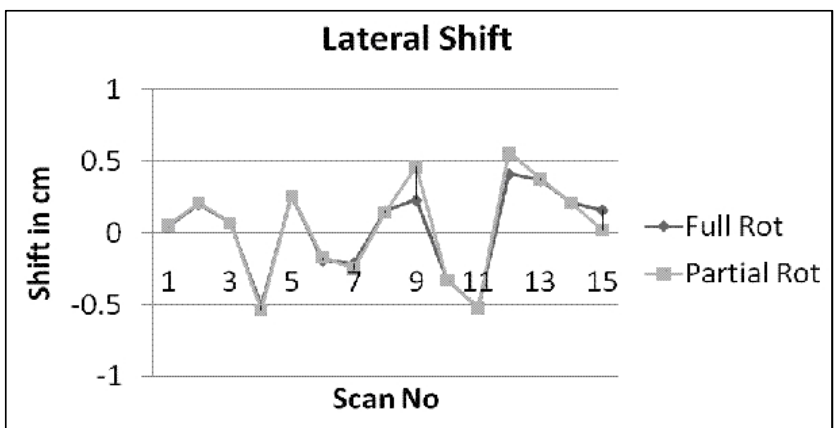

Figure 1: The line graph of lateral shift (X-axis) in breast cases obtained after final matching procedure in full and partial gantry rotation methods.

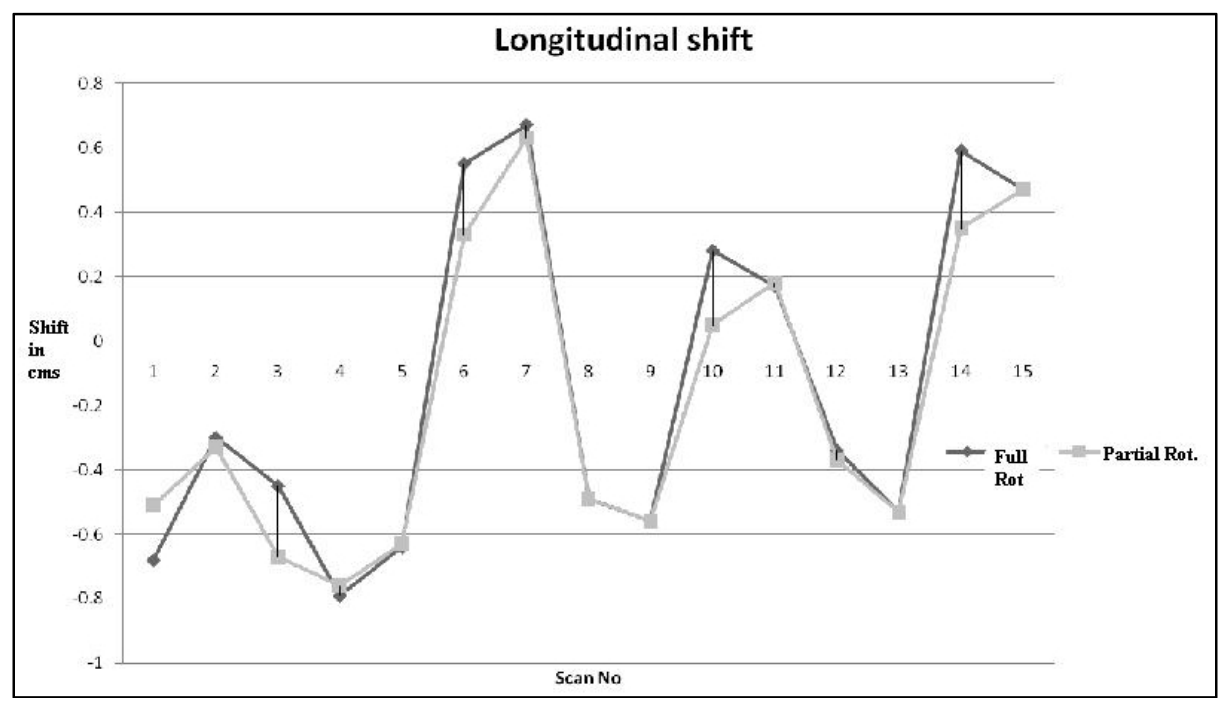

Figure 2: The line graph of longitudinal shift (Y-axis) in breast cases obtained after final matching procedure in full and partial gantry rotations methods. 


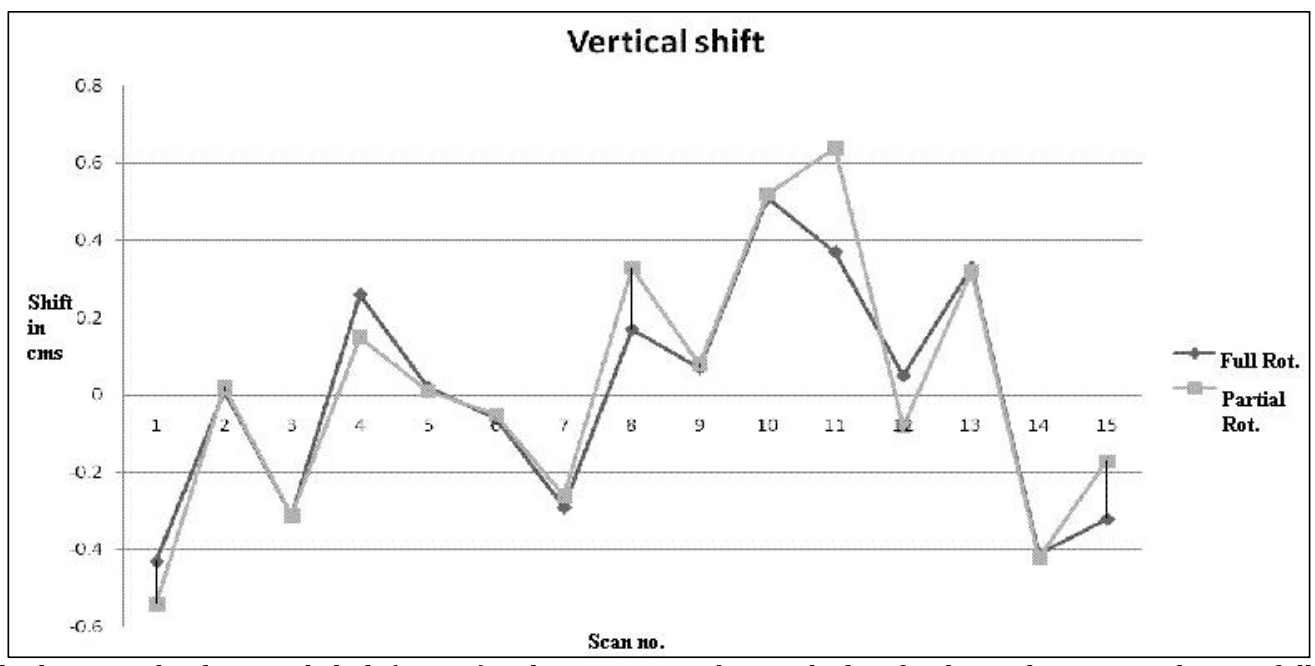

Figure 3: The line graph of vertical shift (Z-axis) in breast cases obtained after final matching procedure in full and partial gantry rotation methods.

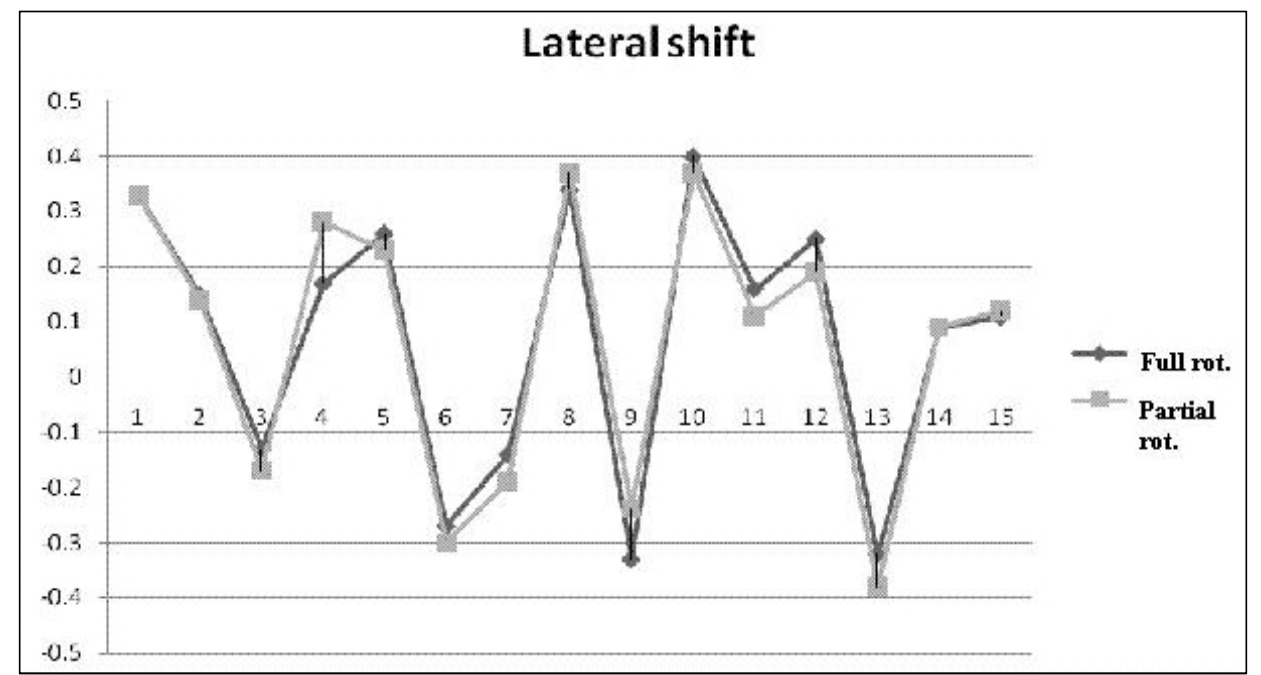

Figure 4: The line graph of lateral shift in head/neck cases obtained after final matching procedure in full and partial gantry rotation methods.

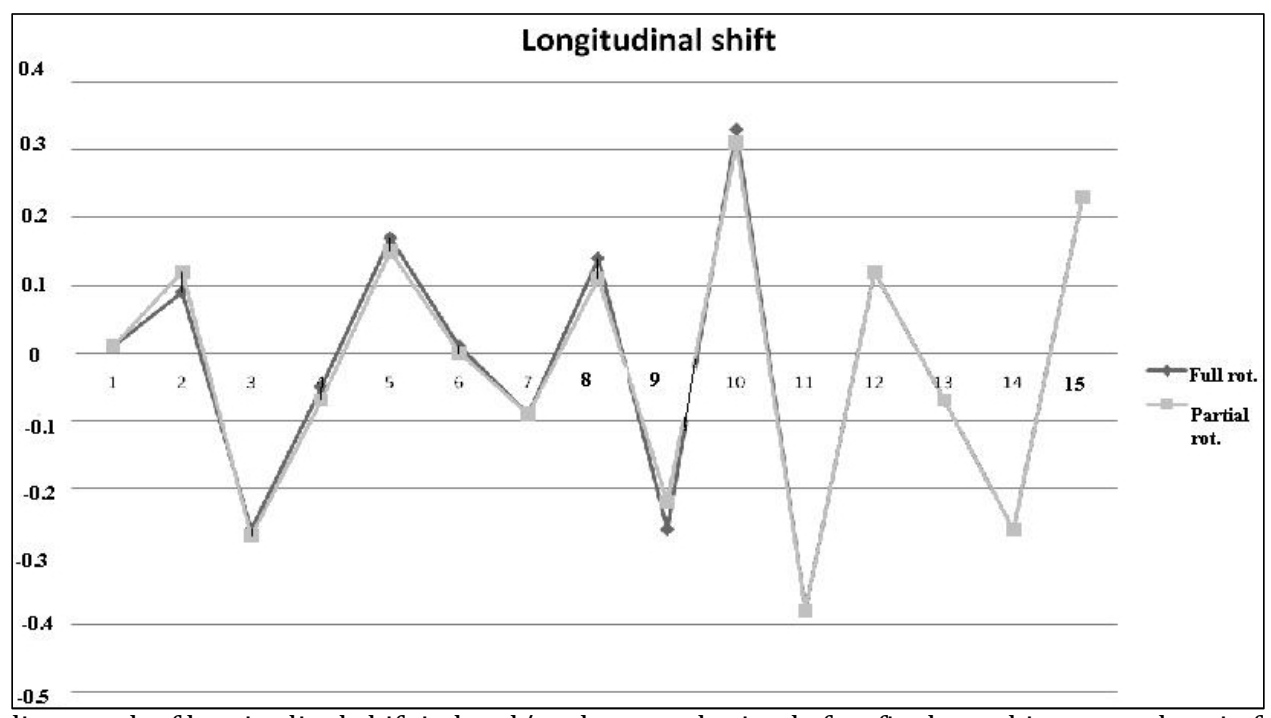

Figure 5: The line graph of longitudinal shift in head/neck cases obtained after final matching procedure in full and partial gantry rotation methods. 


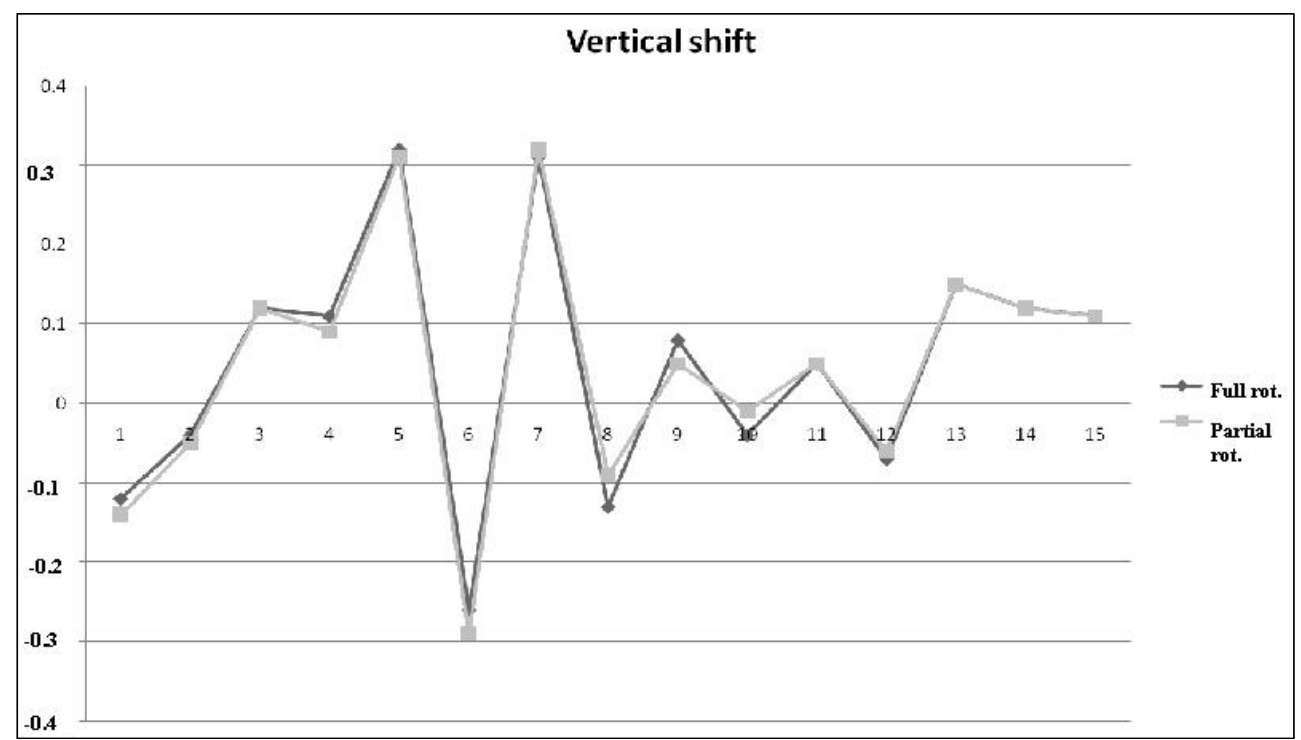

Figure 6: The line graph of vertical shift in head/neck cases obtained after final matching procedure in full and partial gantry rotation methods.

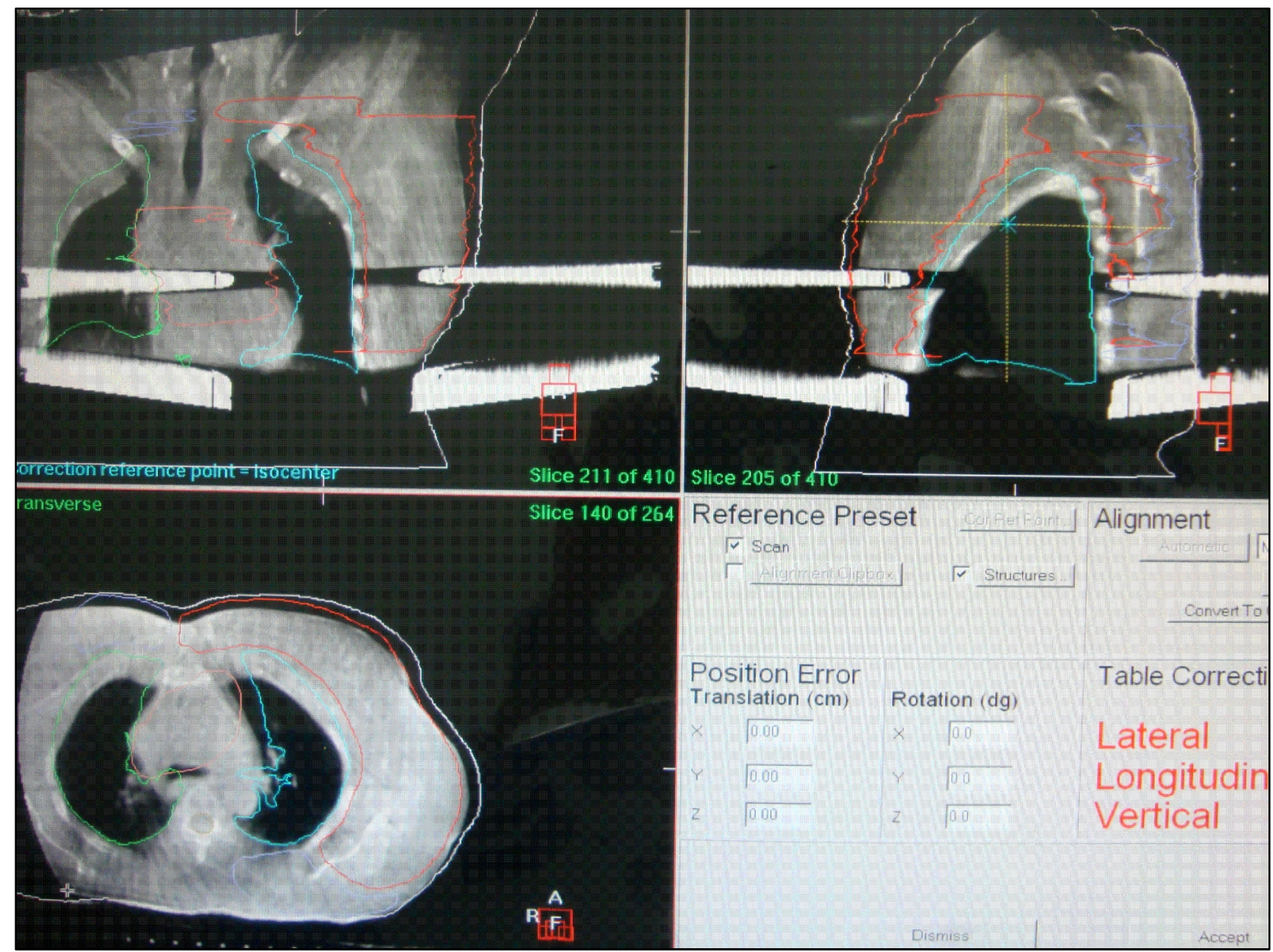

Figure 7: The localization image created in XVI system after obtaining CBCT projections using Full gantry rotation method. It contains all the transverse, sagittal and coronal sections. 


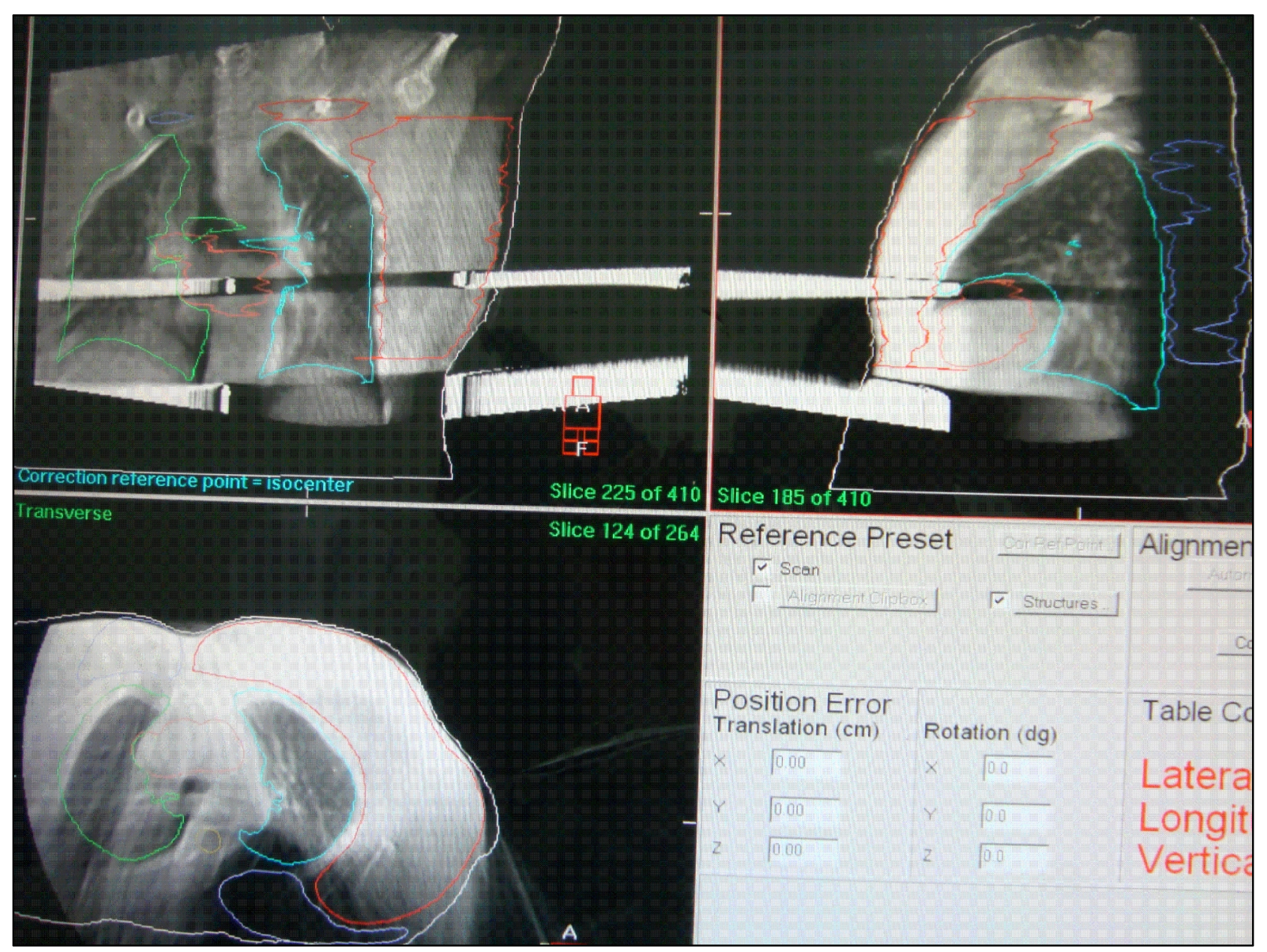

Figure 8: The localization image created in XVI system after obtaining CBCT projections using partial gantry rotation method. It contains all the transverse, sagittal and coronal sections.

Table 1: Rotational shift results in all directions after final matching procedure in full and partial gantry rotational methods in breast cases.

\begin{tabular}{ccccccc}
\hline \hline Scan No & \multicolumn{3}{c}{ Partial rotation } & \multicolumn{3}{c}{ Full rotation } \\
\cline { 2 - 7 } & $\mathrm{X}^{0}$ & $\mathrm{Y}^{0}$ & $\mathrm{Z}^{0}$ & $\mathrm{X}^{0}$ & $\mathrm{Y}^{0}$ & $\mathrm{Z}^{0}$ \\
\cline { 2 - 7 } 1 & 359 & 0.5 & 2.2 & 359.2 & 0.5 & 2.1 \\
2 & 1.1 & 0.3 & 1.9 & 1.1 & 0.4 & 1.9 \\
3 & 2.1 & 359.4 & 0.6 & 2.1 & 359.5 & 0.6 \\
4 & 360 & 0.9 & 357.6 & 359 & 0.9 & 357.4 \\
5 & 359.2 & 1.8 & 0.2 & 359.2 & 1.7 & 0.2 \\
6 & 0.6 & 359.9 & 2.3 & 0.6 & 0.2 & 2.3 \\
7 & 359.4 & 2.1 & 0.3 & 358 & 2.1 & 0.3 \\
8 & 0.9 & 0.5 & 2.5 & 0.9 & 0.3 & 2.4 \\
9 & 1.8 & 358 & 2.2 & 1.8 & 357 & 2.2 \\
10 & 360 & 0.5 & 2.3 & 360 & 0.5 & 2.3 \\
11 & 0.2 & 359.7 & 357.1 & 0.2 & 359.7 & 356.4 \\
12 & 1.6 & 0.6 & 358.7 & 1.6 & 0.6 & 358.9 \\
13 & 357.9 & 1.2 & 0.8 & 358 & 1.2 & 0.8 \\
14 & 0.7 & 0.2 & 359 & 0.7 & 0.2 & 357.9 \\
15 & 358 & 0.2 & 1.4 & 358 & 0.2 & 1.4 \\
\hline \hline
\end{tabular}

Abbreviations: $\mathrm{X}^{0} \overline{\overline{\text { means angular shift in } \mathrm{X} \text { direction; } \mathrm{Y}^{0}}} \overline{\overline{\text { means angular shift in } \mathrm{Y} \text { direction; and } \mathrm{Z}^{0} \text { means }}}$ angular shift in $\mathrm{Z}$ direction 
Table 2: Rotational shift results in all directions after final matching procedure in full and partial gantry rotational methods in head/neck cases.

\begin{tabular}{ccccccc}
\hline \hline Scan No & \multicolumn{3}{c}{ Partial rotation } & \multicolumn{3}{c}{ Full rotation } \\
\cline { 2 - 6 } 1 & $\mathrm{X}^{0}$ & $\mathrm{Y}^{0}$ & $\mathrm{Z}^{0}$ & $\mathrm{X}^{0}$ & $\mathrm{Y}^{0}$ & $\mathrm{Z}^{0}$ \\
\cline { 2 - 6 } 2 & 1.7 & 1.3 & 357 & 1.7 & 1.5 & 357.2 \\
3 & 0.5 & 1 & 1.4 & 0.5 & 1.1 & 1.4 \\
4 & 360 & 2 & 359 & 360 & 1.8 & 359 \\
5 & 360 & 0.9 & 0.1 & 360 & 0.9 & 0.1 \\
6 & 0.1 & 1.1 & 357.8 & 0.1 & 1.1 & 357.9 \\
7 & 1.1 & 1.2 & 0.9 & 0.9 & 1.2 & 0.9 \\
8 & 0.1 & 359 & 1.2 & 0.1 & 359 & 1.2 \\
9 & 359 & 0.8 & 1.7 & 359 & 0.9 & 1.7 \\
10 & 1.6 & 1.1 & 0.1 & 1.9 & 1.1 & 0.3 \\
11 & 1.9 & 0.3 & 359.5 & 1.9 & 0.3 & 359.5 \\
12 & 359.6 & 0.4 & 0.9 & 359.6 & 0.7 & 0.9 \\
13 & 1.1 & 0.8 & 0.5 & 1.7 & 0.8 & 0.1 \\
14 & 0.9 & 359 & 0.7 & 0.9 & 359 & 0.7 \\
15 & 359.9 & 1.2 & 0.3 & 359.9 & 1.2 & 0.5 \\
\hline \hline
\end{tabular}

Abbreviations: $\mathrm{X}^{0} \overline{\overline{\text { means angular shift in } \mathrm{X} \text { direction; } \mathrm{Y}^{0}}} \overline{\text { means angular shift in } \mathrm{Y} \text { direction; and } \mathrm{Z}^{0} \text { means }}$ angular shift in $\mathrm{Z}$ direction

\section{Discussion}

The results from this study revealed that there was very good agreement between shifts of full and partial gantry rotations in both the breast and head and neck cases. Hence, the partial rotation method with less imaging volume is equivalent to full gantry rotation method. As the difference in matching results was negligible between two methods, the one that gives less dose to the patient is typically a better choice. This demonstrated that, although, 3D visual information is slightly inferior with partial rotation, it provided adequate data for matching purpose. The matching procedure is limited to the sub volume defined by using alignment clip box. We generally select the total target volume within the clip box prior to the registration. Therefore, poor reconstruction of the other structures, which are away from the target volume will not affect the registration process, and shift results were similar in both full and partial gantry rotations. It is also found that the reconstructed volume with partial rotation was sufficient to track the anatomy near the target in all selected cases. Our study was primarily focused on the $\mathrm{XVI}$, and other imaging techniques such as electronic portal imaging ${ }^{15,16}$ can also be important in ensuring accurate patient setup during the patient treatment.

\section{Conclusion}

Imaging with partial gantry rotation showed similar results that of full gantry rotation in our selected cases of breast and head and neck cancer using M20 and S20 collimators, respectively. Imaging volume in partial rotation method will be less when compared with full rotation method. Also, time required for imaging and reconstruction using partial gantry rotation will be reduced. Because of these advantages, XVI with partial gantry rotations can potentially be used for the clinical purpose. However, CBCT scan with partial rotation may not always be a correct choice since it depends on the patient thickness and target volume to be scanned. It is suggested that the partial rotation method can be used after verification of CBCT reconstruction in the first fraction of treatment.

\section{Conflict of interest}

The authors declare that they have no conflicts of interest. The authors alone are responsible for the content and writing of the paper.

\section{Acknowledgement}

The authors declare that they have no conflicts of interest. The authors alone are responsible for the content and writing of the paper.

\section{References}

1. Jassal K, Munshi A, Sarkar B, et al. Validation of an integrated patient positioning system: Exactrac and iViewGT on Synergy Platform. Int J Cancer TherOncol. 2014; 2:020212.

2. Alaei P, Spezi E. Commissioning kilovoltage cone-beam CT beams in a radiation therapy treatment planning system. J Appl Clin Med Phys. 2012;13:3971.

3. Sresty NV, Ramanjappa T, Raju AK, et al. Optimal usage of cone beam computed tomography system with different field of views in image guided radiotherapy (IGRT). Int J Cancer Ther Oncol. 2015; 3:334.

4. Feldkamp LA, Davis LC, Kress JW. Practical cone beam Algorithm. J Opt Soc Am A Opt Image Sci Vis. 1984; 1: 612-9. 
5. Jaffray DA, Siewerdsen JH, Wong JW, Martinez AA. Flat-panel cone-beam computed tomography for image-guided radiation therapy. Int J Radiat Oncol Biol Phys. 2002;53:1337-49.

6. Huang Q, Zeng GL, You J, Gullberg GT. An FDK-like cone-beam SPECT reconstruction algorithm for non-uniform attenuated projections acquired using a circular trajectory. Phys Med Biol. 2005;50:2329-39.

7. Cho PS, Johnson RH, Griffin TW. Cone-beam CT for radiotherapy applications. Phys Med Biol. 1995; 40:1863-83.

8. Oldham M, Letourneau D, Watt L, et al. Cone-beam-CT guided radiation therapy: a model for on-line application. Radiother Oncol. 2005; 75: 271-8.

9. Jaffray DA. Emergent technologies for 3-dimensional image-guided radiation delivery. Semin Radiat Oncol. 2005;15:208-16.

10. Yan D, Lockman D, Martinez A, et al. Computed tomography guided management of interfractional patient variation. Semin Radiat Oncol. 2005;15:168-79.

11. Amer A, Marchant T, Sykes J, et al. Imaging doses from the Elekta Synergy X-ray cone beam CT system. Br J Radiol. 2007;80:476-82.
12. Kan MW, Leung LH, Wong W, Lam N. Radiation dose from cone beam computed tomography for image-guided radiation therapy. Int J Radiat Oncol Biol Phys. 2008;70:272-9.

13. McBain CA, Henry AM, Sykes J, et al. X-ray volumetric imaging in image-guided radiotherapy: the new standard in on-treatment imaging. Int J Radiat Oncol Biol Phys. 2006;64:625-34.

14. Meyer J, Wilbert J, Baier K, et al. Positioning accuracy of cone-beam computed tomography in combination with a Hexa POD robot treatment table. Int J Radiat Oncol Biol Phys. 2007;67:1220-8.

15. Rout BK, Shekar MC, Kumar A, Ramesh KKD. Quality control test for electronic portal imaging device using QC-3 phantom with PIPS pro. Int J Cancer Ther Oncol. 2014; 2:02049.

16. Acquah GF, Gustavsson M, Doudoo CO, et al. Clinical use of electronic portal imaging to analyze tumor motion variation during a 3D-conformal prostate cancer radiotherapy using online target verification and implanted markers. Int J Cancer Ther Oncol. 2014; 2:02044. 